\title{
Jacobi's Last Multiplier and Lagrangians for Multidimensional Systems
}

\author{
MC Nucci* and PGL Leach ${ }^{\dagger}$ \\ Dipartimento di Matematica e Informatica \\ Università di Perugia, 06123 Perugia, Italy
}

February 13, 2013

\begin{abstract}
We demonstrate that the formalism for the calculation of the Jacobi last multiplier for a one-degree-of-freedom system extends naturally to systems of more than one degree of freedom thereby extending results of Whittaker dating from more than a century ago and Rao (Proc Ben Math Soc 2 (1940) 53-59) dating from almost seventy years ago. We illustrate the theory with an application taken from the theory of coupled oscillators. We indicate how many Lagrangians can be obtained for such a system.
\end{abstract}

PACS: 02.30.Hq, 02.20.Sv, 45.20.Jj

Keywords: Jacobi's last multiplier, Lie symmetry, Lagrangian.

\section{Introduction}

Jacobi's Last Multiplier is a solution of the linear partial differential equation [3, 4, 5, 6],

$$
\sum_{i=1}^{N} \frac{\partial\left(M a_{i}\right)}{\partial x_{i}}=0,
$$

\footnotetext{
*Email: nucci@unipg.it

${ }^{\dagger}$ permanent address: School of Mathematical Sciences, Westville Campus, University of KwaZulu-Natal, Private Bag X54001 Durban 4000, Republic of South Africa. Email: leachp@ukzn.ac.za, leach@math.aegean.gr
} 
where $\sum_{i=1}^{N} a_{i} \partial_{x_{i}}$ is the vector field of the set of first-order ordinary differential equations for the $N$ dependent variables $x_{i}$, namely

$$
A f=\sum_{i=1}^{n} a_{i}\left(x_{1}, \ldots, x_{n}\right) \frac{\partial f}{\partial x_{i}}=0
$$

or its equivalent associated Lagrange's system

$$
\frac{\mathrm{x}_{1}}{a_{1}}=\frac{\mathrm{x}_{2}}{a_{2}}=\ldots=\frac{\mathrm{x}_{n}}{a_{n}} .
$$

The ratio of any two multipliers is a first integral of the system of first-order differential equations and in the case that this system is derived from the Lagrangian of a one-degree-of-freedom system one has that [5, 6]

$$
\frac{\partial^{2} L}{\partial \dot{q}^{2}}=M
$$

Consequently a knowledge of the multipliers of a system enables one to construct a number of Lagrangians of that system.

We recall that Lie's method [7, 13] for the calculation of the Jacobi Last Multiplier is firstly to find the value of

$$
\Delta=\operatorname{det}\left[\begin{array}{c}
e_{i j} \\
s_{i j}
\end{array}\right],
$$

in which the matrix is square with the elements $e_{i j}$ being the vector field of the set of first-order differential equations by which the system is described and the elements, $s_{i j}$, being the coefficient functions of the number of symmetries of the given system necessary to make the matrix square. If $\Delta$ is not zero, the corresponding multiplier is $M=\Delta^{-1}$. There is an obvious corollary to the results of Jacobi mentioned above. In the case that there exists a constant multiplier, the determinant is a first integral. This result is potentially very useful in the search for first integrals of systems of ordinary differential equations.

The relationship between the Jacobi Last Multiplier and the Lagrangian for a one-degreeof-freedom system is perhaps not widely known although it is certainly not unknown as can be seen from the bibliography in [16. Given a knowledge of a multiplier, (5) gives a simple recipe for the generation of a Lagrangian. The only possible difficulty is the performance of the double quadrature. In this paper we consider that the vector fields of the system of equations and symmetries are known and that we seek the multiplier. From another direction one could know the multiplier and all but one of the symmetries. From (5) the remaining symmetry can be determined [15]. A knowledge of the multipliers of a system enables one to construct a number 
of Lagrangians of that system. Considering the dual nature of the Jacobi Last Multiplier as providing a means to determine both Lagrangians and integrals one is surprised that it has not attracted more attention over the more than one and a half centuries since its introduction. The bibliography of [16] gives a fair indication of its significant applications in the past to which we may add [11], [12], [10], [18], [19], [20], [21].

\section{Theoretical Development}

In Rao [22] there is a development of the work of Whittaker [6] [pp 276-286] on the theory of the Last Multiplier of Jacobi for multidimensional systems. The discussion by Whittaker is limited to dynamical systems without a potential. The work of Rao is restricted to dynamical systems with a Lagrangian which is a quadratic form in the generalised velocities. It does provide a clearer path to an understanding of the methodology than that provided by Whittaker. In particular Rao gives specific formulæ for the equations to be satisfied by the various multipliers. We see in the sequel that a considerable simplification occurs, but we pay due acknowledgement to the inspiration afforded by that paper. Although the theory which we develop here is applicable to dynamical systems of all dimensions, we confine our attention to two dimensions to keep the mathematics as simple as possible so that the essential ideas are quite evident. We assume that we have a Lagrangian, $L\left(t, q_{1}, q_{2}, \dot{q}_{1}, \dot{q}_{2}\right)$, in a standard notation. We write the simplified form of the corresponding Euler-Lagrange equations as

$$
\begin{aligned}
& \ddot{q}_{1}=f_{1}\left(t, q_{1}, q_{2}\right), \\
& \ddot{q}_{2}=f_{2}\left(t, q_{1}, q_{2}\right),
\end{aligned}
$$

where we note that the generalised force is independent of the generalised velocities. The corresponding vector field is

$$
X_{1}=\partial_{t}+\dot{q}_{1} \partial_{q_{1}}+\dot{q}_{2} \partial_{q_{2}}+f_{1} \partial_{\dot{q}_{1}}+f_{2} \partial_{\dot{q}_{2}}
$$

The actual Euler-Lagrange equations are

$$
\begin{aligned}
& \frac{\partial^{2} L}{\partial \dot{q}_{1} \partial t}+\frac{\partial^{2} L}{\partial \dot{q}_{1} \partial q_{1}} \dot{q}_{1}+\frac{\partial^{2} L}{\partial \dot{q}_{1} \partial q_{2}} \dot{q}_{2}+\frac{\partial^{2} L}{\partial \dot{q}_{1}^{2}} f_{1}+\frac{\partial^{2} L}{\partial \dot{q}_{1} \partial \dot{q}_{2}} f_{2}-\frac{\partial L}{\partial q_{1}}=0 \\
& \frac{\partial^{2} L}{\partial \dot{q}_{2} \partial t}+\frac{\partial^{2} L}{\partial \dot{q}_{2} \partial q_{1}} \dot{q}_{1}+\frac{\partial^{2} L}{\partial \dot{q}_{2} \partial q_{2}} \dot{q}_{2}+\frac{\partial^{2} L}{\partial \dot{q}_{1} \partial \dot{q}_{2}} f_{1}+\frac{\partial^{2} L}{\partial \dot{q}_{2}^{2}} f_{2}-\frac{\partial L}{\partial q_{2}}=0
\end{aligned}
$$

in which we have replaced $\ddot{q}_{1}$ and $\ddot{q}_{2}$ by the right sides of the equations of motion above. 
We follow Rao [22] in defining the connection between the last multiplier and the Lagrangian as

$$
M_{i j}=\frac{\partial^{2} L}{\partial \dot{q}_{i} \partial \dot{q}_{j}}, i, j=1,2,
$$

$i e$, we assume the same relationship as for the one-degree-of-freedom case so that, once the multiplier has been calculated, the Lagrangian follows by a double quadrature.

We differentiate (9) and (10) once with respect to both $\dot{q}_{1}$ and $\dot{q}_{2}$. We illustrate the calculation in the case of the differentiation of (9) with respect to $\dot{q}_{2}$. We obtain

$$
\begin{aligned}
& \frac{\partial^{3} L}{\partial \dot{q}_{1} \partial \dot{q}_{2} \partial t}+\frac{\partial^{3} L}{\partial \dot{q}_{1} \partial \dot{q}_{2} \partial q_{1}} \dot{q}_{1}+\frac{\partial^{3} L}{\partial \dot{q}_{1} \partial \dot{q}_{2} \partial q_{2}} \dot{q}_{2} \\
& \quad+\frac{\partial^{2} L}{\partial \dot{q}_{1} \partial q_{2}}+\frac{\partial^{3} L}{\partial \dot{q}_{1}^{2} \partial \dot{q}_{2}} f_{1}+\frac{\partial^{3} L}{\partial \dot{q}_{1} \partial \dot{q}_{2}^{2}} f_{2}-\frac{\partial^{2} L}{\partial \dot{q}_{2} \partial q_{1}}=0,
\end{aligned}
$$

in which we have used the independence of $f_{1}$ and $f_{2}$ from $\dot{q}_{2}$ (in this instance). We use the definition, (11), for $M_{12}$. Then (12) becomes

$$
\begin{aligned}
& \frac{\partial}{\partial t}\left(M_{12}\right)+\frac{\partial}{\partial q_{1}}\left(M_{12} \dot{q}_{1}\right)+\frac{\partial}{\partial q_{2}}\left(M_{12} \dot{q}_{2}\right)+\frac{\partial}{\partial \dot{q}_{1}}\left(M_{12} f_{1}\right) \\
& +\frac{\partial}{\partial \dot{q}_{2}}\left(M_{12} f_{2}\right)+\frac{\partial^{2} L}{\partial \dot{q}_{1} \partial q_{2}}-\frac{\partial^{2} L}{\partial \dot{q}_{2} \partial q_{1}}=0 .
\end{aligned}
$$

In the cases of $M_{11}$ and $M_{22}$ the ultimate and penultimate terms in the equations corresponding to (13) cancel. The other equation for $M_{12}$ has the subscripts reversed so that, when the two equations are added, the terms vanish. Consequently each multiplier is a solution of the equation

$$
\frac{\partial}{\partial t}(M)+\frac{\partial}{\partial q_{1}}\left(M \dot{q}_{1}\right)+\frac{\partial}{\partial q_{2}}\left(M \dot{q}_{2}\right)+\frac{\partial}{\partial \dot{q}_{1}}\left(M f_{1}\right)+\frac{\partial}{\partial \dot{q}_{2}}\left(M f_{2}\right)=0 .
$$

Allowing for the variations in notation (14) is the same equation as (1), ie, the number of degrees of freedom of the system only affects the number of terms in the equation.

\section{Example: A two-dimensional coupled oscillator}

\subsection{Equivalent Lagrangians}

The collinear motion of two particles of equal mass connected to each other by a spring and to fixed points by two other springs of equal spring constants is modelled by the fourth-order 
system 1 .

$$
\begin{aligned}
& \ddot{q}_{1}=-\Omega_{1}^{2} q_{1}-\Omega_{2}^{2}\left(q_{1}-q_{2}\right) \\
& \ddot{q}_{2}=\Omega_{2}^{2}\left(q_{1}-q_{2}\right)-\Omega_{1}^{2} q_{2} .
\end{aligned}
$$

It is evident that a solution of (14) is simply $M=$ constant. We take the constant to be unity for $M_{11}$ and $M_{22}$ and zero for $M_{12}$. Then the corresponding Lagrangian is

$$
L=\frac{1}{2}\left(\dot{q}_{1}^{2}+\dot{q}_{2}^{2}\right)+h_{1}\left(t, q_{1}, q_{2}\right) \dot{q}_{1}+h_{2}\left(t, q_{1}, q_{2}\right) \dot{q}_{2}+h_{3}\left(t, q_{1}, q_{2}\right),
$$

where the three functions, $h_{i}, i=1,3$, are functions of integration. When we substitute $L$, (17), into the general Euler-Lagrange equations, we require that we obtain equations (15) and (16). This requirement imposes the constraints

$$
\begin{aligned}
\frac{\partial h_{1}}{\partial q_{2}}-\frac{\partial h_{2}}{\partial q_{1}} & =0 \\
\frac{\partial h_{1}}{\partial t}-\frac{\partial h_{3}}{\partial q_{1}}-\Omega_{1}^{2} q_{1}-\Omega_{2}^{2}\left(q_{1}-q_{2}\right) & =0 \\
\frac{\partial h_{2}}{\partial t}-\frac{\partial h_{3}}{\partial q_{2}}+\Omega_{2}^{2}\left(q_{1}-q_{2}\right)-\Omega_{1}^{2} q_{2} & =0,
\end{aligned}
$$

where the constraint (18) is common to both equations. The constraint (18) is the two-dimensional version of the condition for the functions to be the divergence of some arbitrary function, $g\left(t, q_{1}, q_{2}\right)$, ie

$$
h_{1}=\frac{\partial g}{\partial q_{1}} \quad \text { and } \quad h_{2}=\frac{\partial g}{\partial q_{2}} .
$$

When (21) is substituted into (19) and (20), one obtains

$$
\begin{gathered}
\frac{\partial^{2} g}{\partial t \partial q_{1}}-\frac{\partial h_{3}}{\partial q_{1}}-\Omega_{1}^{2} q_{1}-\Omega_{2}^{2}\left(q_{1}-q_{2}\right)=0 \\
\frac{\partial^{2} g}{\partial t \partial q_{2}}-\frac{\partial h_{3}}{\partial q_{2}}+\Omega_{2}^{2}\left(q_{1}-q_{2}\right)-\Omega_{1}^{2} q_{2}=0,
\end{gathered}
$$

which can be consistently integrated to give

$$
h_{3}=\frac{\partial g}{\partial t}-\frac{1}{2}\left[\left(\Omega_{1}^{2}+\Omega_{2}^{2}\right)\left(q_{1}^{2}+q_{2}^{2}\right)-2 \Omega_{2}^{2} q_{1} q_{2}\right]+s(t),
$$

\footnotetext{
${ }^{1}$ A simplified version of this system can be found in [14] [pp 479-481]
} 
where $s(t)$ is an arbitrary function which may as well be set at zero, since it makes no contribution to the Euler-Lagrange equation, or included in the other arbitrary function $g\left(t, q_{1}, q_{2}\right)$ which also makes no contribution to the Euler-Lagrange equation.

Eventually we have arrived at the Lagrangian

$$
L=\frac{1}{2}\left(\dot{q}_{1}^{2}+\dot{q}_{2}^{2}\right)+\dot{g}-\frac{1}{2}\left[\left(\Omega_{1}^{2}+\Omega_{2}^{2}\right)\left(q_{1}^{2}+q_{2}^{2}\right)-2 \Omega_{2}^{2} q_{1} q_{2}\right]
$$

ie the arbitrariness in the Lagrangian can be expressed as a total time derivative. Such a Lagrangian has been termed 'gauge variant' [23] and is notable in that the presence of the arbitrary function has no effect upon the number of Noether point symmetries [20]. In this respect it could be regarded as part of the boundary term in the way Noether put it in her formulation of her theorem [24]. The class of Lagrangians described by (25) is an equivalence class.

\section{$3.2 \quad$ A Plethora of Multipliers}

Jacobi proposed to obtain the last multiplier from the solution of the first-order linear partial differential equation, (14). In 1874 Lie [7] showed that one could use point symmetries 2 to determine last multipliers. Lie's method for the calculation of the Jacobi Last Multiplier is firstly to find the value of the determinant in (14). The number of symmetries required depends upon the number of elements in the vector field, which supplies the first row of the matrix, since the matrix must be square. Here we consider that the vector fields of the system of equations and symmetries are known and that we seek the multiplier. The Lie point symmetries of system (15)/16) are

$$
\begin{aligned}
\Gamma_{1} & =q_{2} \partial_{q_{1}}+q_{1} \partial_{q_{2}}+\dot{q}_{2} \partial_{\dot{q}_{1}}+\dot{q}_{1} \partial_{\dot{q}_{2}} \\
\Gamma_{2} & =\exp [Q i t]\left[\left(\partial_{q_{1}}-\partial_{q_{2}}\right)+i Q\left(\partial_{\dot{q}_{1}}-\partial_{\dot{q}_{2}}\right)\right] \\
\Gamma_{3} & =\partial_{t} \\
\Gamma_{4} & =q_{1} \partial_{q_{1}}+q_{2} \partial_{q_{2}}+\dot{q}_{1} \partial_{\dot{q}_{1}}+\dot{q}_{2} \partial_{\dot{q}_{2}} \\
\Gamma_{5} & =\exp [-Q i t]\left(\partial_{q_{1}}-\partial_{q_{2}}-i Q\left(\partial_{\dot{q}_{1}}-\partial_{\dot{q}_{2}}\right)\right] \\
\Gamma_{6} & =\exp \left[i \Omega_{1} t\right]\left[\left(\partial_{q_{1}}+\partial_{q_{2}}\right)+i \Omega_{1}\left(\partial_{\dot{q}_{1}}+\partial_{\dot{q}_{2}}\right)\right] \\
\Gamma_{7} & =\exp \left[-i \Omega_{1} t\right]\left[\left(\partial_{q_{1}}+\partial_{q_{2}}\right)-i \Omega_{1} \Omega_{1} t\left(\partial_{\dot{q}_{1}}+\partial_{\dot{q}_{2}}\right)\right],
\end{aligned}
$$

\footnotetext{
${ }^{2}$ At that time the only symmetries considered were point symmetries. With the extension of the concept of infinitesimal transformations generated by symmetries depending upon derivatives and integrals there has been a natural extension of Lie's valuable contribution to the subject of the Jacobi Last Multiplier.
} 
where $Q=\sqrt{\Omega_{1}^{2}+2 \Omega_{2}^{2}}$, which is the minimal number of Lie point symmetries for an autonomous second-order two-dimensional linear system [25]. Despite the fact that the system possesses only the minimal number of Lie point symmetries there are thirty-five different determinants to be evaluated. Naturally not all of these can be expected to be nonzero or different, but one can easily imagine that this would be the case for a substantial proportion since in the case of a single linear oscillator the number of different multipliers is fourteen out of a possible twenty-eight determinants [20].

It is not our intention to display a large number of multipliers. We simply wish to illustrate two points. The first is the determination of a Lagrangian for the system (15/16) differing from the class represented by (25) which is of a standard form. The second is to display a first integral for the system by taking the ratio of two autonomous multipliers. From the Lagrangian (25) one can construct an autonomous Hamiltonian which is a first integral. Thus we have two first integrals and, as if we did not already know it, the system is integrable from the Theorem of Liouville.

If we take the symmetries $\Gamma_{1}, \Gamma_{2}, \Gamma_{3}$ and $\Gamma_{5}$, the matrix for which the determinant is to be evaluated is

$$
C_{1235}=\left[\begin{array}{lllll}
1 & \dot{q}_{1} & \dot{q}_{2} & -\Omega_{1}^{2} q_{1}-\Omega_{2}^{2}\left(q_{1}-q_{2}\right) & \Omega_{2}\left(q_{1}-q_{2}\right)-\Omega_{1}^{2} q_{2} \\
0 & q_{2} & q_{1} & \dot{q}_{2} & \dot{q}_{1} \\
0 & \exp [Q i t] & -\exp [Q i t] & i Q \exp [Q i t] & -i Q \exp [Q i t] \\
1 & 0 & 0 & 0 & 0 \\
0 & \exp [-Q i t] & -\exp [-Q i t] & -i Q \exp [-Q i t] & i Q \exp [-Q i t]
\end{array}\right] .
$$

We obtain the multiplier

$$
J L M_{1235}=-\frac{1}{2 i Q\left[\left(\dot{q}_{1}+\dot{q}_{2}\right)^{2}+\Omega_{1}^{2}\left(q_{1}+q_{2}\right)^{2}\right]} .
$$

Similarly by taking the symmetries $\Gamma_{1}, \Gamma_{6}, \Gamma_{3}$ and $\Gamma_{7}$ we obtain

$$
J L M_{1637}=\frac{1}{2 \Omega_{2}\left(q_{1}-q_{2}\right)^{2}+\Omega_{1}\left(q_{1}-q_{2}\right)^{2}+\left(\dot{q}_{1}-\dot{q}_{2}\right)^{2}} .
$$

Obviously an integral is

$$
I_{1637 / 1235}=\frac{2 i Q\left[\left(\dot{q}_{1}+\dot{q}_{2}\right)^{2}+\Omega_{1}^{2}\left(q_{1}+q_{2}\right)^{2}\right]}{2 \Omega_{2}\left(q_{1}-q_{2}\right)^{2}+\Omega_{1}\left(q_{1}-q_{2}\right)^{2}+\left(\dot{q}_{1}-\dot{q}_{2}\right)^{2}} .
$$


We can obtain a Lagrangian from $J L M_{1235}$, say, by integrating twice with respect to $\dot{q}_{1}$. We have

$$
\begin{aligned}
L_{1235} & =-\frac{1}{2 i Q \Omega_{1}\left(q_{1}+q_{2}\right)}\left(\dot{q}_{1}+\dot{q}_{2}\right) \arctan \left(\frac{\dot{q}_{1}+\dot{q}_{2}}{\Omega_{1}\left(q_{1}+q_{2}\right)}\right) \\
& +\frac{1}{4 i Q} \log \left[\frac{\Omega_{1}^{2}\left(q_{1}+q_{2}\right)^{2}+\left(\dot{q}_{1}+\dot{q}_{2}\right)^{2}}{\Omega_{1}^{2}\left(q_{1}+q_{2}\right)^{2}}\right]+R_{1}\left(t, q_{1}, q_{2}, \dot{q}_{2}\right) \dot{q}_{1}+R_{2}\left(t, q_{1}, q_{2}, \dot{q}_{2}\right),
\end{aligned}
$$

where $R_{1}$ and $R_{2}$ are functions of integration. When we apply the Euler-Lagrangian condition to (30) and substitute the required equations of motion, (15/16), we obtain the conditions

$$
\frac{i}{2 Q\left(q_{1}+q_{2}\right)}+\left[\Omega_{2}^{2}\left(q_{1}-q_{2}\right)-\Omega_{1}^{2} q_{2}\right] \frac{\partial R_{1}}{\partial \dot{q}_{2}}+\dot{q}_{2} \frac{\partial R_{1}}{\partial q_{2}}-\frac{\partial R_{1}}{\partial t}-\frac{\partial R_{2}}{\partial q_{1}}=0
$$

and

$$
\begin{aligned}
& \frac{i}{2 Q\left(q_{1}+q_{2}\right)}-\left[\Omega_{1}^{2} q_{1}+\Omega_{2}^{2}\left(q_{1}-q_{2}\right)\right] \frac{\partial R_{1}}{\partial \dot{q}_{2}}-\dot{q}_{1} \frac{\partial R_{1}}{\partial q_{1}}-\frac{\partial R_{2}}{\partial q_{2}}+\dot{q}_{1}\left[\Omega_{2}^{2}\left(q_{1}-q_{2}\right)-\Omega_{1}^{2} q_{2}\right] \frac{\partial^{2} R_{1}}{\partial \dot{q}_{2}^{2}} \\
& +\dot{q}_{1} \dot{q}_{2} \frac{\partial^{2} R_{1}}{\partial q_{2} \partial \dot{q}_{2}}+\dot{q}_{1}^{2} \frac{\partial^{2} R_{1}}{\partial q_{1} \partial \dot{q}_{2}}+\dot{q}_{1} \frac{\partial^{2} R_{1}}{\partial t^{2}}=0 .
\end{aligned}
$$

This second equation is a quadratic in $\dot{q}_{1}$ which is not one of the variables in $R_{1}$ and $R_{2}$. The three coefficients give

$$
\begin{aligned}
\frac{\partial^{2} R_{1}}{\partial q_{1} \partial \dot{q}_{2}} & =0 \\
-\frac{\partial R_{1}}{\partial q_{1}}+\left[\Omega_{2}^{2}\left(q_{1}-q_{2}\right)-\Omega_{1}^{2} q_{2}\right] \frac{\partial^{2} R_{1}}{\partial \dot{q}_{2}^{2}}+\dot{q}_{2} \frac{\partial^{2} R_{1}}{\partial q_{2} \partial \dot{q}_{2}}+\frac{\partial^{2} R_{1}}{\partial t^{2}} & =0 \\
\frac{i}{2 Q\left(q_{1}+q_{2}\right)}-\left[\Omega_{1}^{2} q_{1}+\Omega_{2}^{2}\left(q_{1}-q_{2}\right)\right] \frac{\partial R_{1}}{\partial \dot{q}_{2}}-\frac{\partial R_{2}}{\partial q_{2}} & =0 .
\end{aligned}
$$

We note that $R_{2}$ appears only in (31) and (35) and in a very symmetrical way at that. Equations (33) and (34) are easily satisfied by setting $R_{1}$ equal to zero. It then follows that we can take

$$
R_{2}=\frac{i}{2 Q} \log \left(q_{1}+q_{2}\right)
$$




\section{Discussion}

The last multiplier of Jacobi has in the main been sadly neglected. It is evident from the slight literature [6, 22] that its application to systems of more than one degree of freedom was either ignored or misunderstood. In $\S 2$ we saw that there was no difficulty to derive the basic equation for the multiplier. It is the same equation as for the determination of a multiplier for a onedegree-of-freedom system. In the context of our presentation the main implication of that result was that Lie's method was equally applicable to systems of more than one degree of freedom. For the sake of simplicity of presentation we confined our attention to just two dependent variables, but it is quite obvious that the result is general. When we considered the specific example of the system, (15/16), we used obvious solutions of (14) to obtain a class of Lagrangians which includes the usual Lagrangian for such a system. Furthermore we demonstrated the use of the symmetries of the system to construct two other multipliers and hence a second integral of the system 3. Consequently the system, (15/16), is integrable in the sense of Liouville. This is to be expected given that the system is a pair of coupled linear oscillators. In addition to the 'standard' Lagrangiant, (25), we explicitly demonstrated the existence of another Lagrangian. One infers the existence of many.

It is amazing to think that in the case of a two-dimensional linear oscillator, which admits a 15-dimensional Lie point symmetry algebra (the maximum for a two-dimensional system), the possible Lagrangians have to be sought among 1365 different determinants!

Further work is in progress in order to address the case of dissipative systems.

\section{Acknowledgments}

This work was undertaken while PGLL was enjoying the hospitality of Professor MC Nucci and the facilities of the Dipartimento di Matematica e Informatica, Università di Perugia. The continued support of the University of KwaZulu-Natal is gratefully acknowledged.

\section{References}

[1] Jacobi C G J (1842) Sur un noveau principe de la mécanique analytique Comptes Rendus du Académie des Sciences de Paris 15 202-205

\footnotetext{
${ }^{3}$ Obviously the Hamiltonian corresponding to the Lagrangian (25) with the gauge function set at zero gives one integral.

${ }^{4}$ The Lagrangian (25) can be obtained from the matrix $C_{2567}$ which has a constant determinant.
} 
[2] Jacobi CGJ (1844) Sul principio dell'ultimo moltiplicatore, e suo uso come nuovo principio generale di meccanica Giornale arcadico di scienze, lettere e arti Tomo 99 129-146

[3] Jacobi CGJ (1844) Theoria novi multiplicatoris systemati æquationum differentialum vulgarium applicandi: Pars I Journal für Reine und Angewandte Mathematik 27 199-268

[4] Jacobi CGJ (1845) Theoria novi multiplicatoris systemati æquationum differentialum vulgarium applicandi: Pars II Journal für Reine und Angewandte Mathematik 29 213-279 and 333-376

[5] Jacobi CGJ (1886) Vorlesungen über Dynamik. Nebst fünf hinterlassenen Abhandlungen desselben herausgegeben von A Clebsch (Druck und Verlag von Georg Reimer, Berlin)

[6] Whittaker E T (1989) A Treatise on the Analytical Dynamics of Particles and Rigid Bodies (CUP, Cambridge)

[7] Lie S (1874) Veralgemeinerung und neue Verwerthung der Jacobischen Multiplicator-Theorie Fordhandlinger $i$ Videnokabs-Selshabet $i$ Christiania 255-274

[8] Lie S (1912) Vorlesungen über Differentialgleichungen mit Bekannten Infinitesimalen Transformationen (Teubner, Leipzig)

[9] Bianchi L (1918) Lezioni sulla teoria dei gruppi continui finiti di trasformazioni (Enrico Spoerri, Pisa)

[10] Borisov AV, Kilin AA \& Mamaev IS (2005) On a nonholonomic dynamical problem Mathematical Notes 79 734-740

[11] Cosgrove CM (2000) Higher-order Painlevé equations in the polynomial class I. Bureau symbol P2 Studies in Applied Mathematics 104 1-65

[12] Cosgrove CM (2000) Chazy classes IX-XI of third-order differential equations Studies in Applied Mathematics 104 171-228

[13] Lie S (1912) Vorlesungen über Differentialgleichungen mit Bekannten Infinitesimalen Transformationen (Teubner, Leipzig)

[14] Lim Yung-kuo (ed) (2007) Problems and Solutions on Mechanics (World Scientific, Singapore)

[15] Nucci MC \& Leach PGL (2002) Jacobi's last multiplier and the complete symmetry group of the Euler-Poinsot system Journal of Nonlinear Mathematical Physics 9 S-2, 110-121 
[16] Nucci MC (2005) Jacobi last multiplier and Lie symmetries: a novel application of an old relationship Journal of Nonlinear Mathematical Physics 12 284-304

[17] Nucci MC \& Leach PGL (2005) Jacobi's last multiplier and the complete symmetry group of the Ermakov-Pinney equation Journal of Nonlinear Mathematical Physics 12 305-320

[18] Nucci MC (2007) Jacobi last multiplier, Lie symmetries and hidden linearity: "goldfishes" galore Theoretical and Mathematical Physics 151 851-862

[19] Nucci MC \& Leach PGL (2006) Fuchs' solution of Painlevé VI equation by means of Jacobi's last multiplier Journal of Mathematical Physics 48013514 (7 pages)

[20] Nucci MC \& Leach PGL (2007) Lagrangians Galore, arXiv:0706.1008 (submitted)

[21] Nucci MC \& Leach PGL (2007) Gauge variant symmetries for the Schrödinger equation (submitted)

[22] Rao BS Madhava (1940) On the reduction of dynamical equations to the Lagrangian form Proceedings of the Benares Mathematical Society 2 53-59

[23] Lévy-Leblond Jean-Marc (1971) Conservation laws for gauge-variant Lagrangians in Classical Mechanics American Journal of Physics 39 502-506

[24] Noether Emmy (1918) Invariante Variationsprobleme Königlich Gesellschaft der Wissenschaften Göttingen Nachrichten Mathematik-physik Klasse 2 235-267

[25] Gorringe VM \& Leach PGL (1988) Lie point symmetries for systems of second-order linear ordinary differential equations Quœestiones Mathematicœ 11 95-117 